\title{
UN INSTRUMENTO PARA MEDIR LAS COMPETENCIAS PROFESIONALES DE LOS GRADUADOS EN ADMINISTRACIÓN
}

JORGE GUILLERMO ODRIOZOLA CARLOS ESTEBAN BONDAR MABEL GLADYS YANDA MARÍA DE LOS ARCOS MARTÍNEZ

FACULTAD DE CIENCIAS ECONÓMICAS - UNNE

"El gran problema que hay entre la demanda de nuevos profesionales y los universitarios es que cuando estos terminan de estudiar ya no sirven para el mercado. Por eso, hay que abandonar los esquemas de certezas y trabajar con la incertidumbre". 


\title{
RESUMEN
}

partir de los antecedentes internacionales de los proyectos 6x4 UEALC y Tuning América Latina, un equipo de investigadores de la Facultad de Ciencias Económicas de la Universidad Nacional del Nordeste (UNNE) ha desarrollado un cuestionario como instrumento que permite medir la formación recibida por los graduados en administración en términos de competencias al egresar de la carrera, y la aplicación de éstas en el ámbito profesional.

Dicho instrumento surge de un proyecto de investigación acreditado de la UNNE como producto transferible para otras unidades académicas argentinas y del extranjero en virtud de que el listado de competencias genéricas, transversales y específicas expuestas en el 6x4 UEALC fueron definidas como marco para la evaluación de la formación de los egresados de las 61 instituciones de educación superior provenientes de 13 países de América Latina y Europa que participaron en el proyecto internacional.

Como resultado de la aplicación del instrumento a través de procesos de encuestas electrónicas efectuadas sobre la totalidad de los graduados en administración de la UNNE hasta el 15 de septiembre de 2010, se observó que la adquisición de competencias profesionales ha sido en general satisfactoria, pero que requiere de ajustes en determinadas competencias genéricas, transversales y específicas. En particular, resulta necesario ajustar la formación conforme a determinadas demandas del mercado profesional donde se inserta mayoritariamente el egresado, como ser la integración de la organización con el entorno, teniendo en cuenta los aspectos éticos y culturales del medio en el cual desarrolla su gestión.

Palabras clave: formación académica - Licenciatura en Administración - cuestionario encuesta electrónica.

\begin{abstract}
From the international antecedents of the projects 6x4 UEALC and Tuning America Latina, an investigation group of the Economics' Faculty of the Universidad Nacional del Nordeste (UNNE) has developed a questionnaire, as an instrument that allows to measure the administration graduates' formation in terms of competitions after their graduation, and the application of these in the professional area.

Such instrument comes from an accredited research project of the UNNE as transferable product to other academic units from Argentina and abroad in virtue of that the list of generics, transversals and specifics competitions exposed in the $6 \times 4$ UEALC has been defined as frame for the evaluation of the graduates' formation of the 61 high education institute from 13 countries of America Latina and Europe that took part on the international project.
\end{abstract}


As result of the instrument's application through a process of electronics surveys carried out on all the administration graduates of the UNNE until September 15, 2010, was observed that the acquisition of professional competitions has been generally satisfactory, but requires of adjustments in certain generics, transversals and specifics competitions. Especially, it turns out necessary to fit the formation in accordance with certain demands of the professional market where majority of the graduate is inserted, such as the integration of the organization with the environment, considering the ethical and cultural aspects of the environs in which he develops his management.

Keywords: academic formation - Administration Degree - questionnaire - electronic survey.

\section{CONSIDERACIONES INICIALES}

El presente artículo se redactó con la finalidad de dar a conocer al ámbito académico en general, un cuestionario ideado por el equipo de investigación del proyecto acreditado de la Facultad de Ciencias Económicas (Universidad Nacional del Nordeste), denominado "Radiografía y seguimiento del egresado de la Licenciatura en Administración de la Facultad de Ciencias Económicas a partir del plan 1998".

Este cuestionario, basado en el marco de las competencias definidas en el proyecto internacional 6x4 UEALC, constituye un instrumento que permite evaluar la formación de los graduados universitarios en administración al egresar de la carrera y al momento de aplicarla en su profesión. Además, se presentan los resultados finales de las dos mediciones anuales realizadas sobre las opiniones de la totalidad de los egresados en relación a las competencias genéricas, transversales y específicas para el área Administración determinadas por dicho proyecto. Los resultados obtenidos son inéditos, ya que no se conocen mediciones similares para los graduados en Administración de Argentina.

\section{ANTECEDENTES DE LA INVESTIGACIÓN}

El primer antecedente considerado en este trabajo de investigación es el proceso de Bolonia que se inició a partir 1999 con la Declaración de Bolonia, acuerdo que firmaran los ministros de educación de diversos países de Europa.

Los objetivos de este proceso de convergencia fueron adoptar un sistema comparable de títulos que facilite el intercambio y la homologación entre los diferentes países, establecer un sistema internacional de créditos, promover la cooperación europea y la movilidad de estudiantes, docentes, investigadores, y personal de administración y servicios, en pos de la mejora de la calidad de la educación superior y su adaptación a las demandas sociales.

El segundo antecedente, ya de manera más directa, es el Proyecto 6x4 UEALC. 
Este proyecto básicamente latinoamericano, no aspiraba lograr los objetivos planteados en el proceso de Bolonia pero sin embargo, constituye un pilar en el proceso de cambio de la educación superior de América Latina.

El Proyecto 6x4 UEALC fue coordinado por la Asociación Colombiana de Universidades, y participaron 151 profesores investigadores pertenecientes a 61 instituciones de educación superior de nueve países de América Latina y cuatro países de Europa. Su denominación deriva de las seis carreras profesionales: Medicina, Ingeniería Electrónica, Eléctrica y afines, Administración, Matemáticas, Historia y Química; y los cuatro ejes de análisis: créditos académicos, evaluación y acreditación, competencias profesionales, y formación para la innovación y la investigación.

En este proyecto la Universidad Nacional del Nordeste (UNNE) participó con representantes en las carreras de Administración, Ingeniería Electrónica y Química.

Como metodología de investigación se utilizaron fuentes primarias de información a través de la técnica de los Grupos de Enfoque o Focus Groups, tomándose la opinión y percepción de cuatro grupos de actores: estudiantes, profesores, empleadores y graduados universitarios.

Entre los resultados de este proyecto pueden citarse: algunas propuestas de herramientas para la movilidad interinstitucional como el sistema de créditos académicos (SICA) y el complemento al título (CAT), un modelo para la educación y evaluación por competencias (MECO), la elaboración de referentes comunes para la evaluación y la acreditación, y de estrategias de formación para la investigación y la innovación.

Por otra parte, los avances realizados en el eje de las competencias han generado un marco de trabajo común entre los países e instituciones participantes que facilita la comparación de los aprendizajes individuales y el reconocimiento de los perfiles profesionales de las carreras involucradas en el proyecto. Esto a su vez fijó las bases para la construcción de un modelo para la educación y la evaluación de las competencias en América Latina.

El tercer antecedente internacional es el Proyecto ALFA-Tuning-América Latina. En primer término, este proyecto trabajó la metodología Tuning en cuatro áreas del conocimiento: Historia, Ciencias de la Educación, Matemáticas y Ciencias Empresariales, avanzando sobre cuatro líneas de trabajo: competencias genéricas y específicas, métodos de enseñanza, aprendizaje y evaluación, créditos académicos y calidad de los programas.

En una segunda instancia se ampliaron las áreas del conocimiento para el trabajo en profundidad sobre las competencias específicas abarcando: Física, Química, Geología, Enfermería, Derecho, Ingeniería Civil, Medicina y Arquitectura. En este caso, la UNNE sólo participó con un representante en el área de Arquitectura.

La participación en el proyecto abarcó 19 países y 190 universidades latinoamericanas, con el apoyo de más de 200 académicos involucrados. Se utilizó un diseño de muestreo por conglomerados (o muestreo por clusters) con una estructura segmentada o modelo multinivel (nivel individual y de conglomerados países/universidades). Se consultó a grupos de docentes universitarios, graduados, estudiantes y empleadores, a través de las modalidades en línea, 
presencial con reunión explicativa y por correo postal. La diferencia con el Proyecto Tuning europeo fue la inclusión de los estudiantes, que el modelo europeo no contemplaba.

Otros antecedentes más recientes de experiencias de colaboración interinstitucionales e internacionales lo constituyen redes como la Asociación de Universidades del Grupo Montevideo en el MERCOSUR y el Consejo Superior Universitario Centroamericano (CSUCA) para las universidades públicas en Centroamérica. Se destaca la iniciativa conjunta del Proyecto ALFA Puentes entre estas dos redes y otros socios europeos, cuyo objetivo consiste en mejorar los mecanismos de modernización, reforma y armonización de los sistemas de educación superior en América Latina y mejorar las relaciones entre América Latina y Europa en materia de educación superior.

\section{CONCEPTUALIZACIONES SOBRE COMPETENCIAS}

La recopilación de antecedentes realizada en el apartado anterior tiene como punto de convergencia el objetivo de la búsqueda de la mejora de la calidad de la educación superior y su acercamiento a las demandas del mercado y la sociedad en general.

Con este mismo sentido el equipo investigador viene desarrollando este trabajo de investigación teniendo como metas medir el grado de formación adquirida en el transcurso de la carrera en términos de competencias por los graduados en administración de la UNNE, y en qué medida aplican esta formación en la actividad profesional o laboral.

El desarrollo de competencias plantea que además de los conocimientos, la formación académica incluye un cúmulo de actividades y actitudes que la vida en sociedad y el mundo del trabajo exigen a los individuos; y que estas habilidades y actitudes se adquieren a través del desarrollo de competencias.

Resulta entonces necesario realizar una primera conceptualización de este enfoque que como se manifestara incluye conocimientos, habilidades y actitudes relacionadas a una profesión.

Bunk (1994) afirma que: (...) una persona tiene competencia ocupacional si posee los conocimientos, las destrezas y las aptitudes que necesita para desarrollarse en una ocupación, si es capaz de solucionar tareas independientes y flexiblemente. y si tiene la voluntad y la capacidad de desarrollar su esfera de trabajo dentro de la estructura organizacional en la cual se encuentra. (p.9)

Se atribuye el origen del término competencia al lingüista americano Noam Chomsky en el año 1965 a partir de su teoría de la gramática generativa transformacional como competencia lingüística (Chomsky, 1970 citado por Tobón, 2006). Acerca el término al saber hacer en un contexto determinado cuando plantea que la competencia depende del conocimiento que el hablante-oyente tiene de la lengua y de su uso real una situación determinada.

A partir de este planteamiento y con el aporte de otros autores en años posteriores se acuerda en que la definición de competencias es compleja, que incluye tanto la dimensión del saber (capacidades conceptuales o cognitivas), del saber hacer (habilidades instrumentales o 
de procedimiento) y del saber ser que incluye valores y actitudes.

Así la definición de competencias profesionales adoptada por el Proyecto 6x4 UELAC (2008) de manera amplia dice que son las capacidades reales de los egresados de Universidades e Instituciones de Educación Superior, para desempeñar su profesión de manera eficientey eficaz, y se refieren a la aplicación práctica de los conocimientos para el ejercicio profesional. (p.285)

De esta manera, el enfoque adoptado incorpora el concepto de funciones principales de la profesión en la sociedad y la situación donde se lleva a cabo, las acciones para cumplir con la función, el contexto (tecnología, herramientas, etc) en el que se realiza la acción y los criterios de ejecución para identificar el desempeño experto.

\section{DISEÑO DEL INSTRUMENTO PARA MEDIR LAS COMPETENCIAS PROFESIONALES EN LOS GRADUADOS EN ADMINISTRACIÓN}

Tanto el marco conceptual como el listado de competencias definidas en el proyecto 6x4 UEALC permitieron elaborar un cuestionario para captar las opiniones del graduado acerca de la formación adquirida durante el transcurso de su carrera y la aplicada en su ejercicio profesional.

Este cuestionario se diseñó con tres ejes temáticos: competencias genéricas, competencias transversales y competencias específicas de la profesión. En todos los ejes se indagaron acerca de la formación adquirida por el graduado en su paso por la universidad. Y en el caso particular de las competencias específicas, se incorporó un sub-eje con preguntas referidas a las aplicadas en el ejercicio profesional. El desarrollo y análisis de cada uno de estos ejes se presenta en el punto 9.

\section{ESCALA DE MEDICIÓN UTILIZADA}

Para medir el grado de formación adquirida y aplicada en la profesión se utilizó la escala definida en el Proyecto Tuning América Latina.

Esta escala recoge las opiniones a través de cuatro descriptores:

- Nada, equivalente numéricamente a 1.

- Poco, equivalente numéricamente a 2.

- Bastante, equivalente numéricamente a 3.

- Mucho, equivalente numéricamente a 4.

Esta escala expone en su valor más alto el ideal a alcanzar en cuanto a formación académica del graduado (Mucho, equivalente a 4). Para esta investigación, un valor de 2,5 (valor medio de la escala) permitió identificar aquellas competencias donde la formación de los graduados ha sido deficiente'

\footnotetext{
${ }^{1}$ Este valor depende de la expectativa mínima de formación que cada unidad académica se plantee como objetivo frente a sus graduados. En esta investigación se tomó 2,5 por considerarse un valor equidistante de los extremos de la escala.
} 


\section{MÉTODO DE RECOPILACIÓN DE DATOS}

$\mathrm{Al}$ mes de mayo de 2011 se efectuaron dos censos ${ }^{2}$ remitiendo una versión electrónica autoadministrable del cuestionario a los graduados. El proyecto de investigación tiene ejecución desde el 2009 al 2012, restando realizar dos mediciones aún, que posiblemente tengan similar comportamiento a las opiniones de los $95^{3}$ graduados encuestados.

\section{PROCEDIMIENTO PARA CALCULAR EL GRADO DE FORMACIÓN ADQUIRIDA EN LA CARRERA Y APLICADA EN LA PROFESIÓN DE LOS GRADUADOS}

Los resultados se obtuvieron mediante la aplicación de la media aritmética sobre la totalidad de las respuestas recibidas.

En el caso particular de las competencias específicas adquiridas durante el transcurso de la carrera, la cual constó de grupos de preguntas para la medición de cada competencia, se calculó la media de las medias aritméticas en cada grupo de preguntas.

Posteriormente, para obtener el valor global de las competencias genéricas, transversales y específicas, se procedió a calcular la media de las medias aritméticas individuales.

\section{ANÁLISIS DE LOS RESULTADOS}

En los resultados individuales, cada competencia en particular se comparó con el valor medio de la escala, y por otro lado, en las competencias específicas, se compararon las adquiridas en la carrera con las aplicadas en la profesión con el objeto de detectar posibles diferencias entre ambas.

En los resultados globales, se compararon por un lado, las competencias genéricas, transversales y especificas adquiridas durante el transcurso de la carrera entre sí y respecto del valor medio de la escala ${ }^{4} ; y$ por el otro, las competencias específicas adquiridas en la carrera frente a la importancia asignada a las mismas por los graduados en su desempeño laboral. El $98 \%$ de los graduados encuestados se encuentran trabajando al momento de responder el cuestionario.

\section{EJES TEMÁTICOS DE LAS COMPETENCIAS PROYECTADAS EN EL INSTRUMENTO DE MEDICIÓN}

\subsection{Eje Temático de las Competencias Genéricas.}

Competencias genéricas son aquellas que permiten una interacción en sociedad como personas capaces de compartir y trabajar interdisciplinariamente, y por ende son las que deben compartir profesionales de todas las disciplinas, incluyendo a los administradores. (Informe Final Proyecto 6x4 UELAC, 2008, p. 232)

\footnotetext{
${ }^{2}$ Se contactó a la totalidad de los egresados que culminaron sus estudios al 15 de setiembre de cada año.

${ }^{3}$ Representan el $96 \%$ del universo.

${ }^{4} 2,5$.
} 
El glosario de la Organización Internacional del Trabajo (OIT, 2002) señala que estas competencias son adquiridas en el período escolar y en la práctica del trabajo. sirven para cualquier actividad profesional y son apoyadas en bases científicas y tecnológicas y en atributos humanos, tales como creatividad, condiciones intelectuales y capacidad de transferir conocimientos a nuevas situaciones. Cita como ejemplos las competencias para la toma de decisión, iniciativa, la empatía y la simpatía, la habilidad numérica y computacional, la habilidad verbal y de conversación.

A partir de esta conceptualización y con el listado de las 14 competencias genéricas definidas en el proyecto 6x4 UEALC (ver anexo III), se diseñó la primera sección del cuestionario, como puede apreciarse parcialmente en el Cuestionario 1 :

\author{
Cuestionario 1: Imagen parcial del Cuestionario no 1 \\ Proyecto de seguimiento a los graduados en \\ Administración de la Universidad Nacional del \\ Nordeste \\ 'Obligalono \\ COMPETENCIAS GENËRICAS EN EL GRADUADO \\ Son aquellas que comparten profesionalas de dwergas disciplinas \\ EVALÚE INDICANDO DEL 1 AL A SIENDO LOE DESCPIPTORES DE LA ESCALA 1 = NADA 2 \\ - POCO, 3 = BASTANTE. 4 = MUCHD \\ DURANTE EL CURSADO DE SU CARRERA, HA RECIBIDO FORMACION \\ PARA: \\ 1. Buscar, seleccionar, utilizar y evaluar la información actualizada y pertinente para la \\ toina de decisiones en su campo prolesional \\ $\begin{array}{llll}1 & 2 & 3 & 4\end{array}$ \\ Nada 0000 Mucho \\ 2. Utilizar tecnologias de información y comunicación genéricas y especializadas en su \\ campo como soporte de su ejercicio profesional \\ 1234 \\ Nada 0000 Mucho \\ 3. Idenılficar, dalimitar y resolvar sltuaciones y/a prohlamas " \\ $\begin{array}{llll}1 & 2 & 3 & 4\end{array}$ \\ Nada 0000 Mucho
}

Las opiniones de los graduados en Administración de la Universidad Nacional del Nordeste fueron en promedio las siguientes (figura 1): 
Figura 1: Formación adquirida en las competencias genéricas

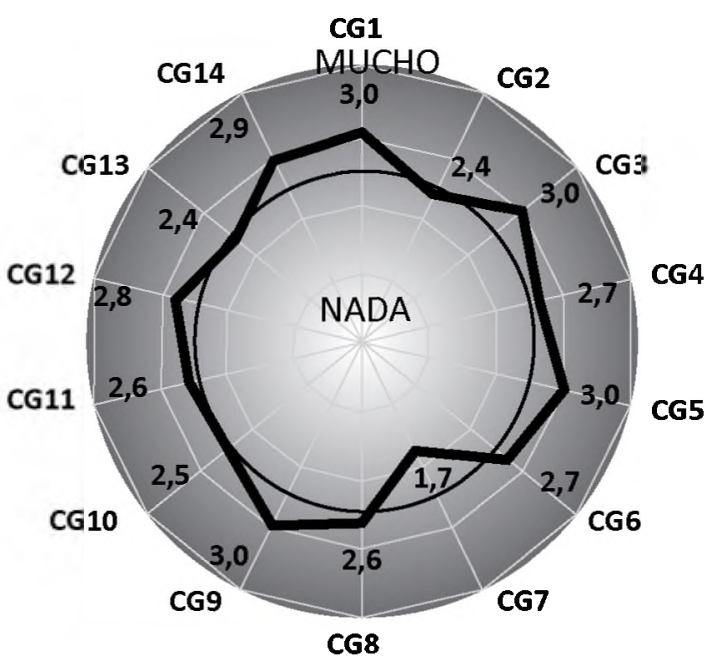

Se observa que las competencias genéricas CG2, CG7 y CG13 exponen valores por debajo de 2,5 .

\subsection{Eje temático de las competencias transversales}

Las competencias transversales se definen en el Proyecto 6x4 UELAC (2008) como aquellas que habilitan para generar acciones efectivas de manera compartida con personas que tienen lenguajes comunes en su desempeño profesional, es decir, son aquellas que comparten las personas de disciplinas similares (p. 245).

La OIT (2002) enumera como ejemplos de estas competencias el dominio de un procesador de textos que sirve para todas las profesiones que necesiten la competencia de la escritura, las nociones de la estadística fundamental necesarias en muchas profesiones, nociones de ventas importantes en un gran número de profesiones del sector de servicios y la lectura de la partitura musical indispensable en aquellas profesiones que están ligadas a la música.

Para esta sección, el cuestionario constó de una pantalla (Cuestionario 2) que indagó sobre la formación adquirida en cada una de las 8 competencias transversales definidas por el proyecto 6x4 UEALC (ver listado en anexo IV): 
Cuestionario 2: Imagen parcial del Cuestionario $\mathbf{N}^{\circ} 2$

Proyecto de seguimiento a los graduados en Administración de la Universidad Nacional del Nordeste

"Obligatorio

COMPETENCIAS TRANSVERSALES EN EL GRADUADO

Compartidas por disciplinas similares o personas que tienen lenguajes comunes en el desempeño profesional

EVALUE INDICANDO DEL 1 AL 4 SIENDO LOS DESCRIPTORES DE LA ESCALA 1 = NADA 2 $=$ POCO 3 = BASTANTE. $4=$ MUCHO

DURANTE EL CURSADO DE SU CARRERA, HA RECIBIDO FORMACIÓN PARA:

1. Comprender y aplicar los elementos basicos de la Administración de organizaciones, sus diversas áreas de acción y la interacción entre las mismas

$$
\begin{array}{llll}
1 & 2 & 3 & 4
\end{array}
$$

Nada 0000 Mucho

2. Comprender y aplicar los principios de la economia tanto a nivel macro como micro, explicando can claridad los fenómenos de interacción económica en la sociedad y en las organlzaciones *

$\begin{array}{llll}1 & 2 & 3 & 4\end{array}$

Pada 0000 ruscho

Las opiniones de los graduados en Administración de la Universidad Nacional del Nordeste fueron en promedio las siguientes (figura 2):

Figura 2: Formación adquirida en las competencias transversales

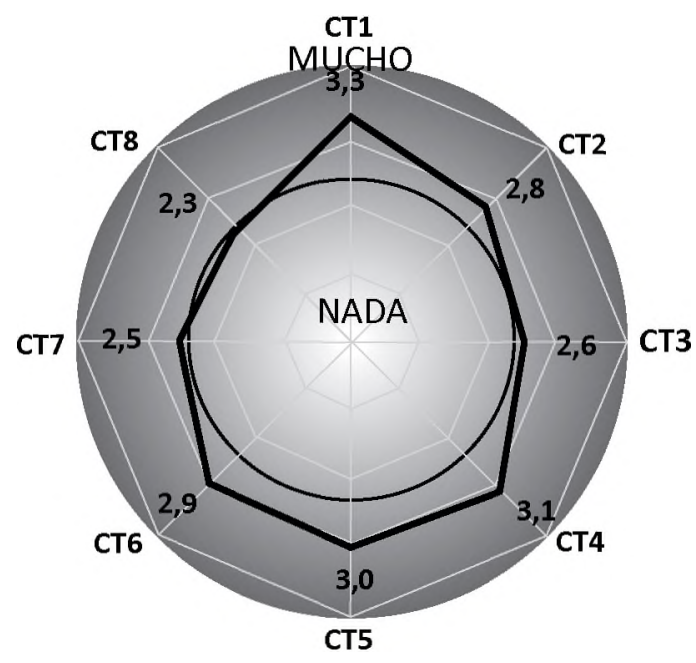

Se observa que la competencia transversal CT8 tiene su valor por debajo de 2,5. 


\subsection{Eje temático de las competencias específicas}

\subsubsection{Formacion adquirida en competencias específicas durante el transcurso de la carrera}

Las competencias específicas agrupan todas las relacionadas directamente con el quehacer profesional, y normalmente son el objeto directo de análisis en los procesos de definición de competencias en las diversas disciplinas y carreras (Informe Final Proyecto 6x4 UELAC, 2008, p. 254).

Según Huerta Amezola, Pérez García y Castellanos Castellano (2000) son la base particular del ejercicio profesional y están vinculadas a condiciones específicas de ejecución.

Por su parte el glosario de la OIT (2002) plantea que las competencias profesionales son aquellas adquiridas en la especialización profesional. que no pueden ser transferibles, a no ser indirectamente, por las habilidades adquiridas que puedan ser readaptadas y que los contenidos son ligados estrictamente a una especialidad definida.

En este sub-eje. las 18 competencias definidas por el Proyecto 6x4 UEALC (ver listado en anexo V) se midieron a través de los grupos de preguntas referidas a las evidencias de la formación adquirida por el graduado en aspectos específicos de la profesión (Cuestionario 3). Estos grupos de preguntas pueden hallarse en el documento del citado proyecto $6 \times 4$ UEALC.

\section{Cuestionario 3: Imagen parcial del Cuestionario No 3}

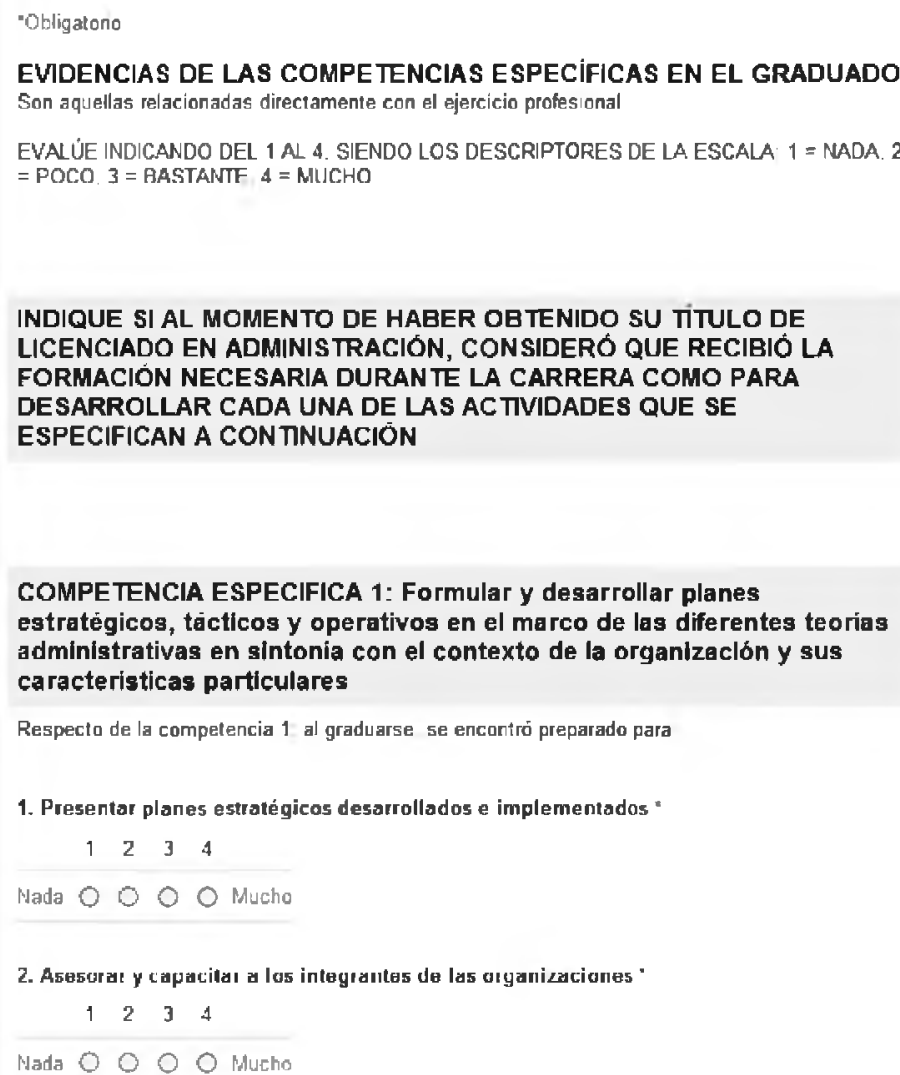


Las opiniones de los graduados en Administración de la Universidad Nacional del Nordeste fueron en promedio las siguientes (figura 3):

Figura 3: Formación adquirida en las competencias específicas

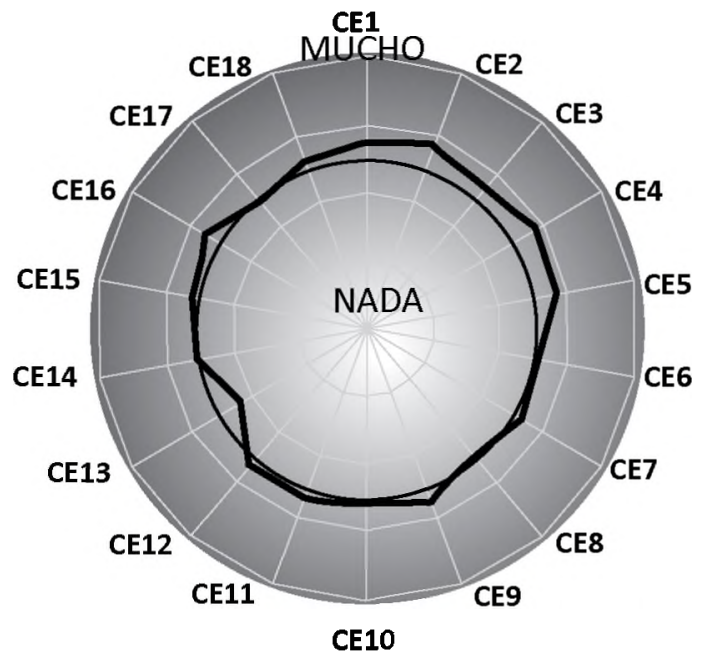

Se observa que las competencias específicas CE13 y CE17 exponen valores por debajo de 2,5.

9.3.2 Aplicación de las competencias específicasen el desempeño profesional

Con el propósito de evaluar y comparar la formación adquirida y aplicada en la profesión, se incorporó una pantalla (Cuestionario 4) para recopilar esta opinión de los graduados:

\section{Cuestionario 4: Imagen parcial del Cuestionario No 4}

Proyecto de seguimiento a los graduados en Administración de la Universidad Nacional del Nordeste

'Obligatorio

IMPORTANCIA DE LAS COMPETENCIAS ESPECIFICAS DEL GRADUADO

EN LA PROFESIÓN

EVALÚE INDICANDO DEL 1 AL 4 SIENDO LOS DESCRIPTORES DE LA ESCALA: 1 = NADA 2 $=$ POCO 3 = BASTANTE. 4 = MUCHO

INDIQUE LA IMPORTANCIA QUE TIENE PARA US TED CADA COMPETENCIA EN SU DESEMPEN̈O LABORAL, ES DECIR, EN QUÉ MEDIDA LAS UTILIZA EN SU TRABAJO.

1. Formular y desarrollar planes estratégicos, tácticos y operativos en el marco de las diferentes teorias administrativas en sintonia con el contexto de la organización y sus caracteristicas particulares

$$
\begin{array}{llll}
1 & 2 & 3 & 4
\end{array}
$$

Nada 0000 Mucho

2. Propiciar sinergias que permitan enfocar la gestión al logro de los resultados esperados, alineando las áreas funclonales de la organización

$\begin{array}{llll}1 & 2 & 3 & 4\end{array}$

Nada 0000 Mucho 
Las opiniones de los graduados en Administración de la Universidad Nacional del Nordeste fueron en promedio las siguientes (figura 4):

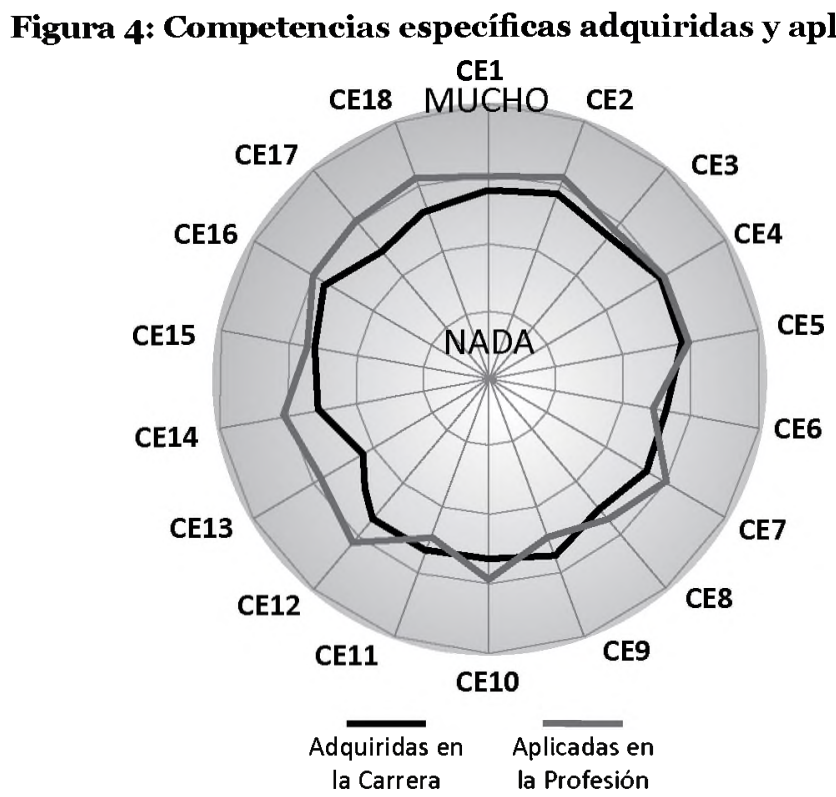

$\mathrm{Al}$ analizar las competencias específicas, pueden compararse las adquiridas en la carrera y las utilizadas en el desempeño profesional. De esta comparación surgen:

- Competencias específicas donde la formación adquirida es superior al grado de aplicación de las mismas en las actividades laborales: CE6, CE9 y CE11 (3 de 18 competencias).

- Competencias específicas donde el grado de aplicación es superior a la formación adquirida en la carrera: CE1, CE2, CE3, CE4, CE5, CE7, CE8, CE10, CE12, CE13, CE14, CE15, CE16, CE17 y CE18 (15 de 18 competencias).

\section{RESULTADOS GENERALES DE LA INVESTIGACIÓN}

Los resultados obtenidos para las competencias genéricas, transversales y específicas fueron los siguientes (figura 5):

Figura 5: Formación adquirida en las distintas competencias

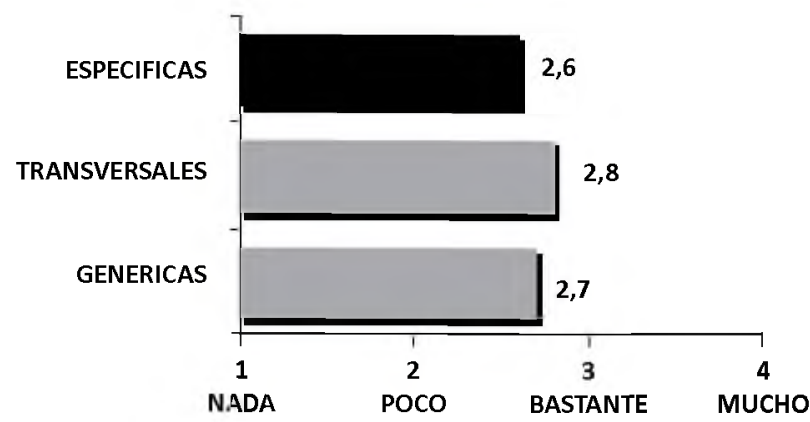


Asimismo, agregando los valores de las competencias específicas aplicadas en la profesión se pueden comparar las demandas del mercado profesional en cuanto a formación requerida y la suministrada por la unidad académica (figura 6):

Figura 6: Competencias específicas

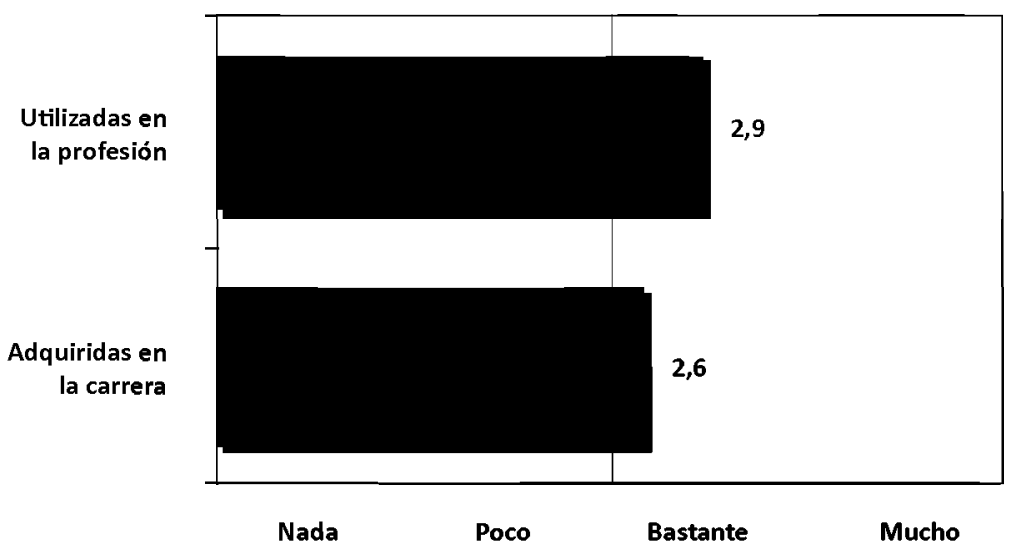

\section{CONCLUSIONES}

En los resultados de la investigación efectuada pudo determinarse si los graduados tuvieron deficiencias en su formación.

En este sentido, se han obtenido valores inferiores a la media de la escala aplicada en tres competencias genéricas, en una competencia transversal y en dos competencias específicas.

Por otra parte, de la comparación entre las competencias adquiridas durante la carrera y las aplicadas en la profesión se detectaron quince en las que el mercado profesional demanda mayor formación en el egresado.

Globalmente, las opiniones de los graduados demostraron que han recibido una formación satisfactoria al finalizar su carrera.

En otro término, el cuestionario desarrollado ha resultado un instrumento funcional, de lectura sencilla, rápido acceso, fácil administración y de utilidad para la medición de la percepción de los graduados respecto de las competencias adquiridas a lo largo de la formación académica.

Esta herramienta será posible transferirla a otras unidades académicas en las que se dicten la carrera de administración, ya que los listados y definiciones de competencias contempladas han sido extraídas del Proyecto 6x4 UEALC en el que han participado instituciones de diferentes países latinoamericanos, entre ellas, la Universidad Nacional del Nordeste.

Los resultados de esta medición son de suma importancia para el análisis curricular y la modificación de los planes de estudio. En particular, el plan de estudios de la carrera de Licenciatura en Administración de la Facultad de Ciencias Económicas cuenta con 10 años de antigüedad, motivo por el cual actualmente se está realizando una revisión de su diseño para propiciar una currícula más acorde a las demandas del mercado. 


\section{ANEXOS}

12.1 ANEXO I: Sección completa del cuestionario que mide las 14 competencias genericas definidas a partir del proyecto $6 \times 4$ UEALC

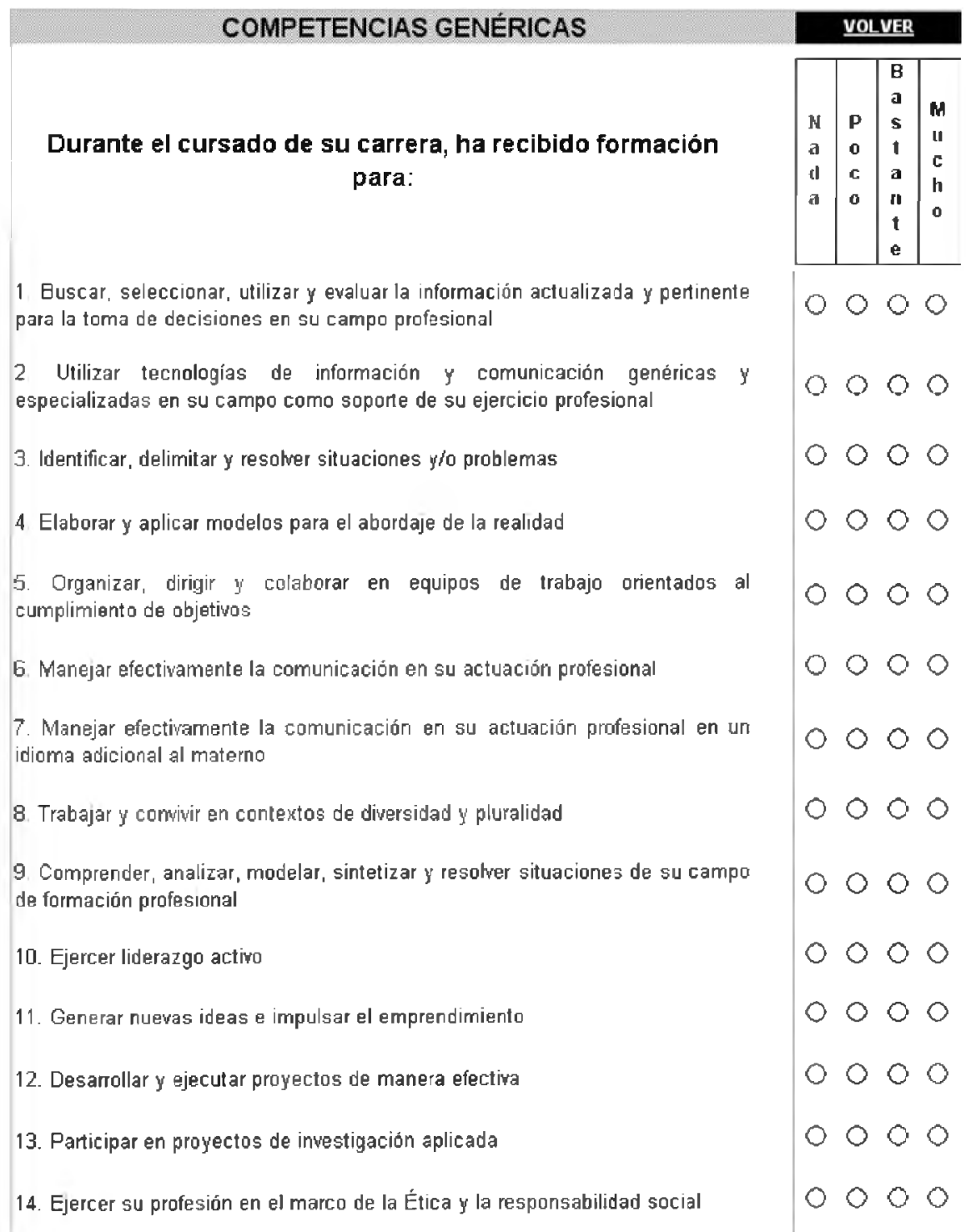




\subsection{ANEXO II: Sección completa del cuestionario que mide las 8 competencias transversales definidas a partir del proyecto $6 \times 4$ UEALC}

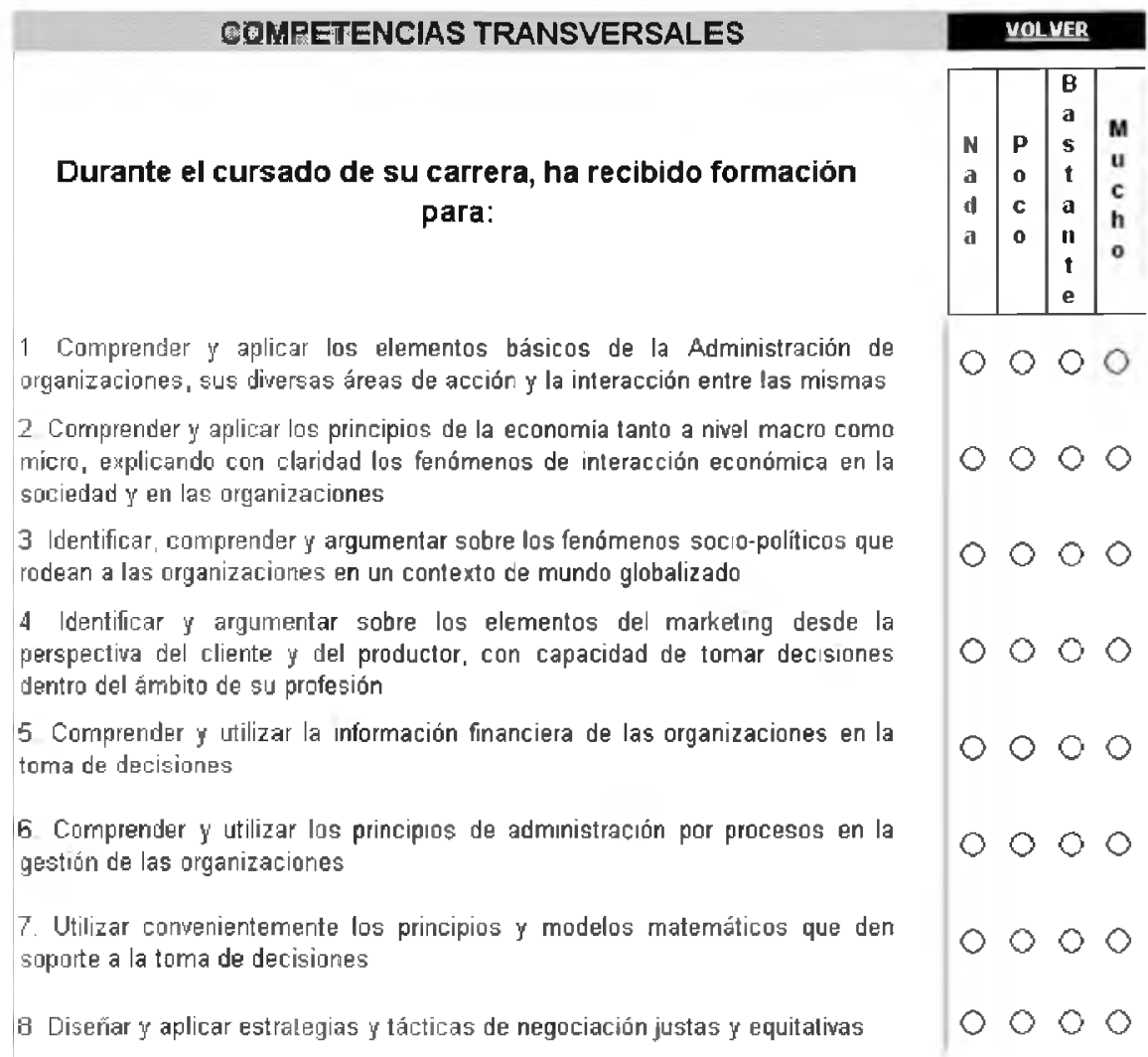

\subsection{ANEXO III: Listado de competencias genéricas}

1. Buscar, seleccionar, utilizar y evaluar la información actualizada y pertinente para la toma de decisiones en su campo profesional.

2. Utilizar tecnologías de información y comunicación genéricas y especializadas en su campo como soporte de su ejercicio profesional.

3. Identificar, delimitar y resolver situaciones y/o problemas.

4. Elaborar y aplicar modelos para el abordaje de la realidad.

5. Organizar. dirigir y colaborar en equipos de trabajo orientados al cumplimiento de objetivos.

6. Manejar efectivamente la comunicación en su actuación profesional.

7. Manejar efectivamente la comunicación en su actuación profesional en un idioma adicional al materno.

8. Trabajar y convivir en contextos de diversidad y pluralidad. 
9. Comprender, analizar, modelar, sintetizar y resolver situaciones de su campo de formación profesional.

10. Ejercer liderazgo activo.

11. Generar nuevas ideas e impulsar el emprendimiento.

12. Desarrollar y ejecutar proyectos de manera efectiva.

13. Participar en proyectos de investigación aplicada.

14. Ejercer su profesión en el marco de la Ética y la responsabilidad social.

\subsection{ANEXO IV: Listado de competencias transversales}

1. Comprender y aplicar los elementos básicos de la Administración de organizaciones, sus diversas áreas de acción y la interacción entre las mismas.

2. Comprender y aplicar los principios de la economía tanto a nivel macro como micro, explicando con claridad los fenómenos de interacción económica en la sociedad y en las organizaciones.

3. Identificar, comprender y argumentar sobre los fenómenos sociopolíticos que rodean a las organizaciones en un contexto de mundo globalizado.

4. Identificar y argumentar sobre los elementos del marketing desde la perspectiva del cliente y del productor, con capacidad de tomar decisiones dentro del ámbito de su profesión.

5. Comprender y utilizar la información financiera de las organizaciones en la toma de decisiones.

6. Comprender y utilizar los principios de administración por procesos en la gestión de las organizaciones.

7. Utilizar convenientemente los principios y modelos matemáticos que den soporte a la toma de decisiones.

8. Diseñar y aplicar estrategias y tácticas de negociación justas y equitativas.

\subsection{ANEXO V: Listado de competencias específicas}

1. Formular y desarrollar planes estratégicos, tácticos y operativos en el marco de las diferentes teorías administrativas en sintonía con el contexto de la organización y sus características particulares.

2. Propiciar sinergias que permitan enfocar la gestión al logro de los resultados esperados, alineando las áreas funcionales de la organización.

3. Diseñar, implementar y evaluar procesos de comercialización que tengan como eje central a los diferentes tipos de clientes y/o demandantes de servicios que interactúan con la organización.

4. Identificar y evaluar la viabilidad de oportunidades de negocios, procesos, productos y servicios. 
5. Identificar, diseñar e implementar procesos de negocio y/o prestación de servicios orientados a la optimización de los resultados de la organización.

6. Administrar los sistemas logísticos y productivos integrales que impacten en la cadena de valor.

7. Diseñar y gestionar sistemas de seguimiento y evaluación de los objetivos planteados en el direccionamiento estratégico.

8. Integrar al componente administrativo y empresarial el marco jurídico pertinente.

9. Gestionar integralmente proyectos empresariales aplicables a diferentes organizaciones.

10. Tomar decisiones de inversión, financiamiento y gestión de recursos (materiales y humanos) a partir del análisis de los sistemas de información (internosexternos).

11. Orientar la organización a la creación de valor a partir de modelos de valoración y riesgo que sean de alto impacto en las decisiones empresariales.

12. Liderar y administrar el talento humano para el logro y consecución de los objetivos de la organización.

13. Integrar la organización con el entorno, teniendo en cuenta los aspectos éticos y culturales del medio en el cual desarrolla su gestión.

14. Optimizar la gestión empresarial apoyado en sistemas de información efectivos y en el uso de las tecnologías de la información y la comunicación (TIC's).

15. Analizar, diseñar y sostener sistemas de calidad y de la gestión del cambio.

16. Desarrollar la cultura de la organización en el marco de la misión, visión y valores que la identifican y diferencian.

17. Crear, mantener y desarrollar redes organizacionales e interpersonales para la consecución de los objetivos.

18. Diseñar, rediseñar e implementar estructuras, procedimientos y sistemas alineados a las estrategias organizacionales.

\section{REFERENCIAS BIBLIOGRÁFICAS}

Bunk, G. (1994). La transmisión de las competencias en la formación y perfeccionamiento profesionales de la RFA. Revista Europea de Formación Profesional. (1) pp. 8-14..

Certificación de competencias profesionales. Glosario de términos técnicos. (2002). Proyecto Avance Conceptual y Metodológico de la Formación Profesional en el Campo de la Diversidad en el Trabajo y de la Certificación Profesional. Oficina Internacional del Trabajo (OIT) y Secretaría de Políticas Públicas de Empleo del Ministerio de Trabajo y Empleo (MTE) de Brasil. Organizadores: Alexim, J. C. \& Brígido, R. Colaboradora: Freire, L. Consultado el 25/11/2010 en http: www.oei.es/etp/certificacion_competencias_profesionales_glosario.pdf 
Huerta Amezola, J., Pérez García, I. y Castellanos Castellano, A. (2000). Desarrollo curricular por competencias profesionales integrales. Revista digital Educar, (13) Consultado el 26/10/2010 en http://educar.jalisco.gob.mx/13/13Huerta.html

Informe Final del Proyecto 6x4 Unión Europea, América Latina y el Caribe UEALC (2008). Propuestas y acciones universitarias para la transformación de la educación superior en América Latina. Bogotá: Asociación Colombiana de Universidades (ASCUN) En http://www.6x4uealc.org/site2008/po1/6x4_po1c.pdf

Informe Final del Proyecto Tuning - América Latina 2004-2007 (2007). Reflexiones $y$ perspectivas de la Educación Superior en América Latina. Bilbao: Universidad de Deusto. En http://www.tuning.unideusto.org/tuningal/

Tobón, S. (2006). Formación basada en competencias: pensamiento complejo, diseño curricular y didáctica. Bogotá: Ecoe Ediciones.

\section{CURRÍCULUM VITAE JORGE GUILLERMO ODRIOZOLA}

Máster en Dirección de Marketing en la Empresa, Profesor Titular y Vicedecano - Facultad de Ciencias Económicas, Universidad Nacional del Nordeste - Argentina

godriozola@eco.unne.edu.ar

\section{CARLOS ESTEBAN BONDAR}

Licenciado en Administración y Contador Público, Auxiliar Docente de Primera - Facultad de Ciencias Económicas, Universidad Nacional del Nordeste - Argentina

cebondar@eco.unne.edu.ar

\section{MABEL GLADYS YANDA}

Especialista en Docencia Universitaria, Auxiliar Docente de Primera - Facultad de Ciencias Económicas, Universidad Nacional del Nordeste - Argentina

\section{mabel@unne.edu.ar}

\section{MARÍA DE LOS ARCOS MARTÍNEZ}

Máster en Política y Gestión Universitaria, Profesora Adjunta y Secretaría Académica - Facultad de Ciencias Económicas, Universidad Nacional del Nordeste - Argentina

mamartinez@eco.unne.edu.ar 